\title{
Supervised Local Subspace Learning for Region Segmentation and Categorization in High-Resolution Satellite Images
}

\author{
Yen-wei Chen ${ }^{1,2}$ and Xian-hua Han ${ }^{1,2}$ \\ ${ }^{1}$ Elect \& Inf. Eng. School, Central South Univ. of Forest and Tech., Changsha, China \\ chen@is.ritsumei.ac.jp \\ ${ }^{2}$ Graduate School of Science and Engineering, Ritsumeikan University, Japan
}

\begin{abstract}
We proposed a new feature extraction method based on supervised locality preserving projections (SLPP) for region segmentation and categorization in high-resolution satellite images. Compared with other subspace methods such as PCA and ICA, SLPP can preserve local geometric structure of data and enhance within-class local information. The generalization of the proposed SLPP based method is discussed in this paper.
\end{abstract}

Keywords: supervised locality preserving projections, region segmentation, categorization, high-resolution satellite images, subspace learning, independent component analysis, generalization.

\section{Introduction}

Recently several high resolution satellites such as IKONOS, Quickbird have been launched and the high resolution images $(1 \mathrm{~m})$ are available. Region segmentation and categorization in high-resolution satellite images are important issues for many applications, such as remote sensing (RS) and geographic information system (GIS) updating. The satellite image is a record of relative reflectance of particular wavelengths of electromagnetic radiation. A particular target reflection depends on the surface feature of the target and the wavelength of the incoming radiation. Multi-spectral information has been widely used for classification of remotely sensed images [1]. Since the spectra are combined by many factors such as object reflectance and instrumentation response, there are strong correlations among the spectra. Principal component analysis (PCA) has been proposed to reduce the redundancy among the spectra and find efficient representation for classifications or segmentations [2]. In our previous works, we proposed to apply independent component analysis (ICA) to learn the efficient spectral representation [3]. Since ICA features are higher-order uncorrelated while PCA features are second-order uncorrelated, higher classification performance has been achieved by ICA. Though ICA is a powerful method for finding efficient spectra 
representation, it is an unsupervised approach and it lacks the local geometric structure of data.

Locality preserving projections (LPP) was proposed to approximate the eigenfunctions of the Laplace Beltrami operator on the image manifold, and be applied for face recognition and image indexing [4]. In this paper, we propose a new approach based on supervised locality preserving projections (SLPP) for classification of highresolution satellite images. The scheme of the proposed method is shown in Fig.1. The observed multi-spectral images are first transformed by SLPP and then the transformed spectral components are used as features for classifications. A probabilistic neural network (PNN) [5] is used as a classifier. Compared with other subspace methods such as PCA and ICA, SLPP can not only find the manifold of images but also enhance the within-class local information. In our previous work, the proposed method has been successfully applied to IKONOS images and experimental results show that the proposed SLPP based method outperforms ICA-based method [6]. In this paper, we discuss the generalization of the proposed SLPP based method. We use only one image as training sample for SLPP subspace learning and classifier (PNN) training. The trained SLPP subspace and PNN are used for other test image segmentation and categorization.

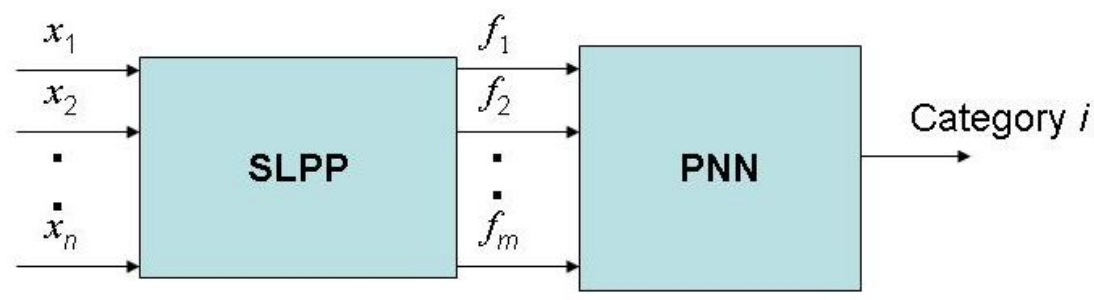

Fig. 1. The proposed method based on SLPP

The paper is organized as following: the supervised LPP for feature extractions is presented in Sec.2, the probabilistic neural network for classifications is presented in Sec. 3 and the experimental results are shown in Sec.4. Finally, the conclusion is given in Sec.5.

\section{Supervised Locality Preserving Projections (SLPP)}

The problem of subspace learning for image for feature extraction is the following. Given a set of spectral feature vectors $\mathbf{x}_{1}, \mathbf{x}_{2}, \cdots, \mathbf{x}_{m}$ in $\mathbf{R}^{n}$ of images, the goal is to find an efficient representation $\mathbf{f}_{i}$ of $\mathbf{x}_{i}$ such that $\left\|\mathbf{f}_{i}-\mathbf{f}_{j}\right\|$ reflects the neighborhood relationship between $\mathbf{f}_{i}$ and $\mathbf{f}_{j}$. In other word, if $\left\|\mathbf{f}_{i}-\mathbf{f}_{j}\right\|$ is small, then $\mathbf{x}_{i}$ and $\mathbf{x}_{j}$ are belong to same class. Here, we assume that the images reside on a sub-manifold embedded in the ambient space $\mathbf{R}^{n}$. 
LPP seeks a linear transformation $\mathbf{P}$ to project high-dimensional data into a lowdimensional sub-manifold that preserves the local Structure of the data. Let $\mathbf{X}=\left[\mathbf{x}_{1}, \mathbf{x}_{2}, \cdots, \mathbf{x}_{m}\right]$ denote the feature matrix whose column vectors is the sample feature vectors in $\mathbf{R}^{n}$. The linear transformation $\mathbf{P}$ can be obtained by solving the following minimization problem:

$$
\min _{\mathbf{P}} \sum_{i j}\left(\mathbf{P}^{T} \mathbf{x}_{i}-\mathbf{P}^{T} \mathbf{x}_{j}\right)^{2} B_{i j}
$$

where $B_{\mathrm{ij}}$ evaluate the local structure of the image space. In this paper, we use normalized correlation coefficient of two sample as the penalty weight if the two sample belong to the same class:

$$
B_{i j}=\left\{\begin{array}{lc}
\frac{\mathbf{x}_{i}^{T} \mathbf{x}_{j}}{\sum_{l=1}^{n} \mathbf{x}_{i l}^{2} \sum_{l=1}^{n} \mathbf{x}_{j l}^{2}} & \text { if sample } i \text { and } j \text { are in same class } \\
0 & \text { otherwise }
\end{array}\right.
$$

By simple algebra formulation, the objective function cam be reduced to:

$$
\begin{aligned}
& \frac{1}{2} \sum_{i j}\left(\mathbf{P}^{T} \mathbf{x}_{i}-\mathbf{P}^{T} \mathbf{x}_{j}\right)^{2} B_{i j} \\
& =\sum_{i} \mathbf{P}^{T} \mathbf{x}_{i} D_{i i} \mathbf{P}^{T} \mathbf{x}_{i}-\sum_{i j} \mathbf{P}^{T} \mathbf{x}_{i} B_{i j} \mathbf{P}^{T} \mathbf{x}_{j} \\
& =\mathbf{P}^{T} \mathbf{X}(\mathbf{D}-\mathbf{B}) \mathbf{X}^{T} \mathbf{P}=\mathbf{P}^{T} \mathbf{X} \mathbf{L} \mathbf{X}^{T} \mathbf{P}
\end{aligned}
$$

$\mathbf{D}$ is a diagonal matrix; its entries are column (or row, since B is symmetric) sum of $\mathbf{B}$, $D_{i i}=\sum_{j} B_{i j} . \mathbf{L}=\mathbf{D}-\mathbf{S}$ is the Laplacian matrix. Then, the linear transformation $\mathbf{P}$ can be obtained by minimizing the objective function under constraint:

$$
\mathbf{P}=\underset{\mathbf{P}^{T} \mathbf{X D X} \mathbf{X}^{T} \mathbf{P}=1}{\arg \min } \mathbf{P}^{T} \mathbf{X}(\mathbf{D}-\mathbf{B}) \mathbf{X}^{T} \mathbf{P}
$$

Finally, the minimization problem can be converted to solving a generalized eigenvalue problem as follows:

$$
\mathbf{X L X}^{T} \mathbf{P}=\lambda \mathbf{X D X}^{T} \mathbf{P}
$$

\section{Probabilistic Neural Network (PNN)}

The PNN model is based on Parzen's results on probability density function (PDF) estimators [5]. PNN is a three-layer feedforward network consisting of input layer, a pattern layer, and a summation or output layer as shown in Fig.2. We wish to form a Parzen estimate based on $K$ patterns each of which is $n$-dimensional, randomly sampled from $c$ classes. The PNN for this case consists of $n$ input units comprising the input layer, where each unit is connected to one and only one $f$ the c category units. The connection from the input to pattern units represents modifiable weights, which 
will be trained. Each category unit computes the sum of the pattern units connected to it. A radial basis function and a Gaussian activation are used for the pattern nodes.

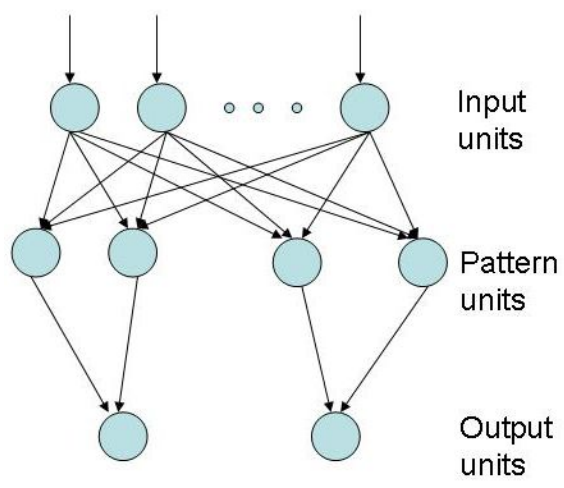

Fig. 2. PNN architecture

The PNN is trained in the following way. First, each pattern (sample feature) $\mathbf{f}$ of the training set is normalized to have unit length. The first normalized training pattern is placed on the input units. The modifiable weights linking the input units and the first pattern unit are set such that $\mathbf{w}_{1}=\mathbf{f}_{1}$. Then, a single connection from the first pattern unit is mage to the category unit corresponding to the known class of that pattern. The process is repeated with each of the remaining training patterns, setting the weights to the successive pattern units such that $\mathbf{w}_{k}=\mathbf{f}_{k}$ for $k=1,2, \cdots, K$. After such training we have a network which is fully connected between input and pattern units, and sparsely connected from pattern to category units. The trained network is then used for segmentation and categorization in the following way. A normalized test pattern $\mathbf{f}$ is placed at the input units. Each pattern unit computes the inner product to yield the net activation $\mathbf{y}$,

$$
y_{k}=\mathbf{w}_{k}^{T} \cdot \mathbf{f}
$$

and emits a nonlinear function of $y_{k}$; each output unit sums the contributions from all pattern units connected to it. The activation function used is $\exp \left(\left\|\mathbf{x}-\mathbf{w}_{k}\right\| / \delta^{2}\right)$. Assuming that both $\mathbf{x}$ and $\mathbf{w}_{k}$ are normalized to unit length, this is equivalent to using $\exp \left(\|\mathbf{x}-1\| / \delta^{2}\right)$.

\section{Experimental Results}

The proposed method has been apply to classification of IKONOS images. IKONOS simultaneously collects one-meter resolution black-and-white (panchromatic) images and four-meter resolution color (multi-spectral) images. The multi-spectral images 
consist of four bands in the blue $(B)$, green $(G)$, red $(R)$ and near-infrared wavelength regions. And the multi-spectral images can be merged with panchromatic images of the same locations to produce "pan-sharpened color" images of 1-m resolution. In our experiments, we use only RGB spectral images for region segmentation and categorization. Two typical IKONOS color images as shown in Fig.4(a) and Fig.5(a) are used in our experiments. One shown in Fig.4(a) is used as sample image for learning and another one shown in Fig.5(a) is used as test image for testing. In our experiments, we define 5 categories: sea, forest, ground, road and others. We first randomly selected 100 points from each category. In order to keep some texture information, we use a sub-block of $3 \times 3$ surround the selected point and the RGB values the sub-blocks are used as spectral feature vector $\mathbf{x}$ with a dimension of 27. $\mathbf{x}_{i}(i=1,2, \cdots, 5 \times 100)$ are used to learn the SLPP subspace for feature extraction and train the probabilistic neural network for region segmentation and categorization. The learning and training process is shown in Fig.3. It is a two-step learning process. We first use $\mathbf{x}$ to learn the SLPP subspace $\mathbf{P}$. Then the projection of $\mathbf{f}=\mathbf{P}^{T} \mathbf{x}$ is used as inputs of PNN for training of PNN, which are also used as features for region segmentation and categorization.

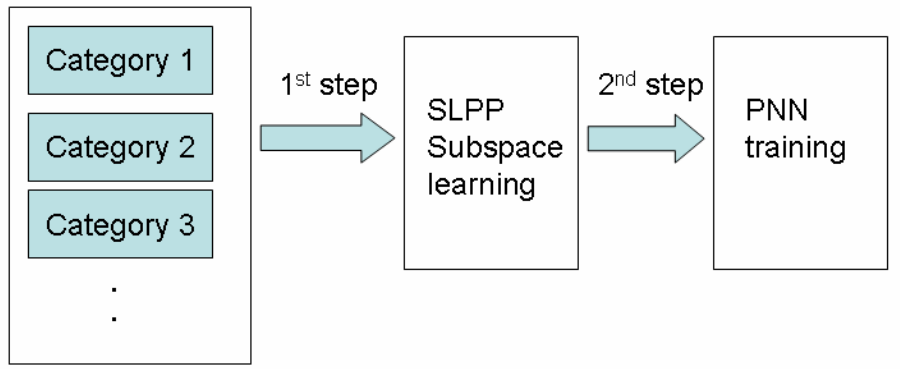

Sample images

Fig. 3. Two-step learning process

Once the SLPP subspace and PNN are trained, they are used for feature extraction and region segmentation, respectively. The segmentation and categorization process is just as shown in Fig.1. The feature vector $\mathbf{x}(27 \times 1)$ of each pixel is first projected to the SLPP subspace and the projection is input to trained PNN. The output of PNN is the index of category. Thus the satellite image can be segmented into 5 regions and each region is categorized. The region segmentation and categorization results for sample image (Fig.4(a)) are shown in Fig.4(b)-4(f). The results for test image (Fig.5(a)) are shown in Fig.5(b)-5(f). It can be seen that we can get a satisfy segmentation result for the sample image (Fig.4), while test image (Fig.5) the result is not very satisfied. For example, a part of sea was categorized into the forest region as shown in Fig.5(c). Since only one image is used as training sample in our experiments, the generalization of the PNN is very limited. The segmentation and categorization accuracy will be improved by increasing the number of sample images. 


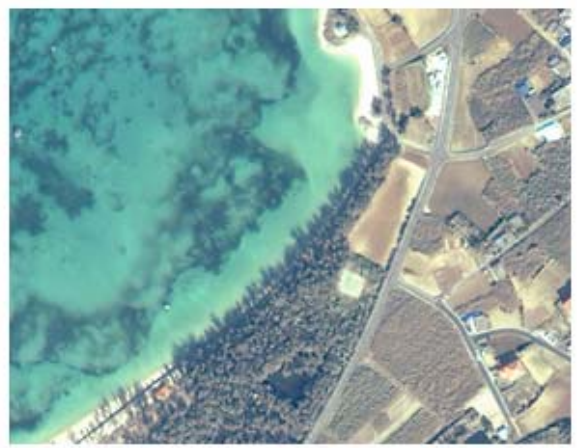

(a) IKONOS image

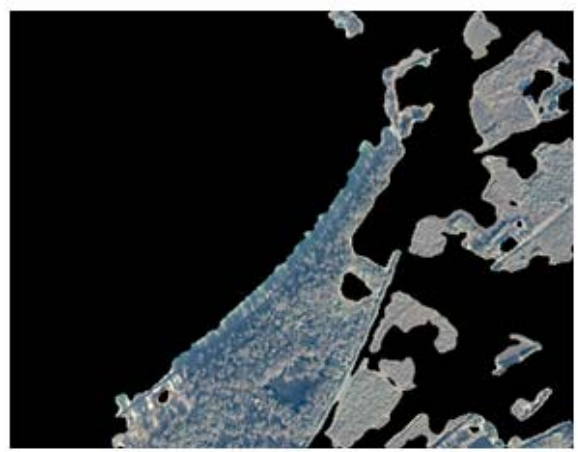

(c) Forest

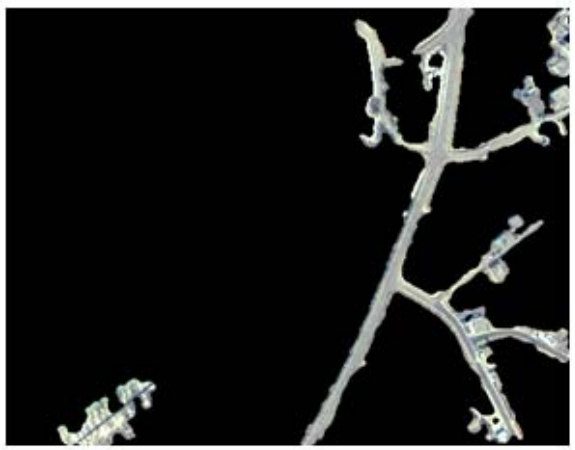

(e) Road

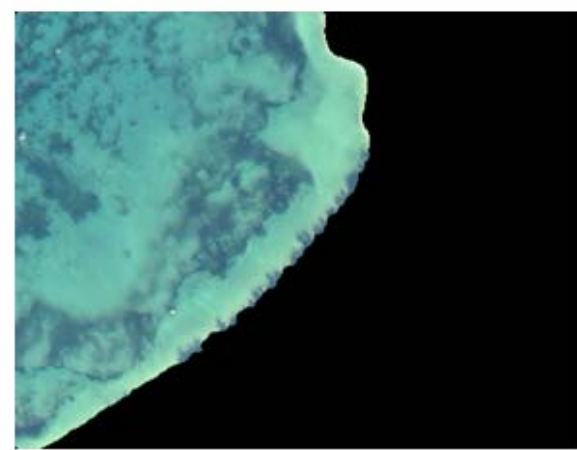

(b) Sea

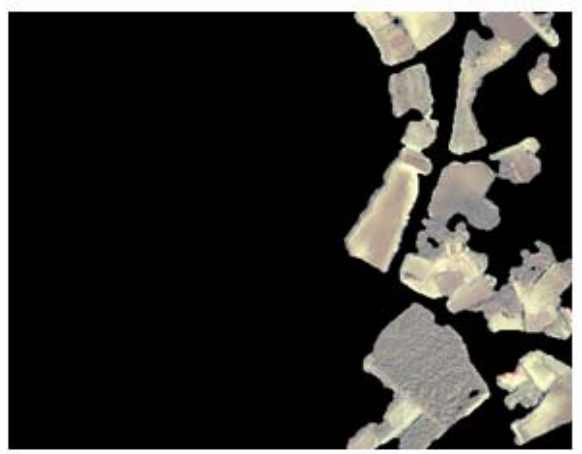

(d) Ground

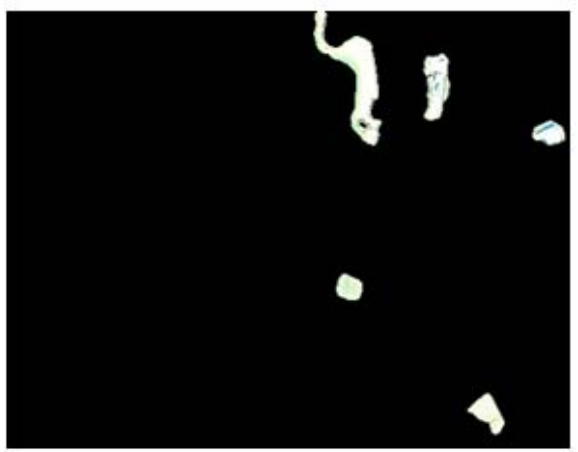

(f) Others

Fig. 4. Region segmentation and categorization results (sample image) (IKONOS image: Copyright (C) 2003 Japan Space Imaging Corporation) 


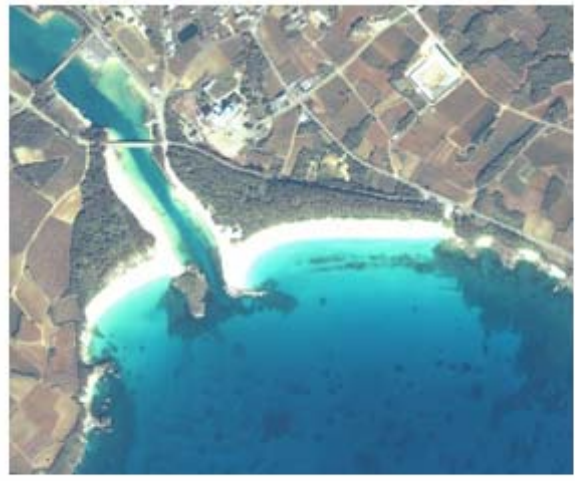

(a) IKONOS image

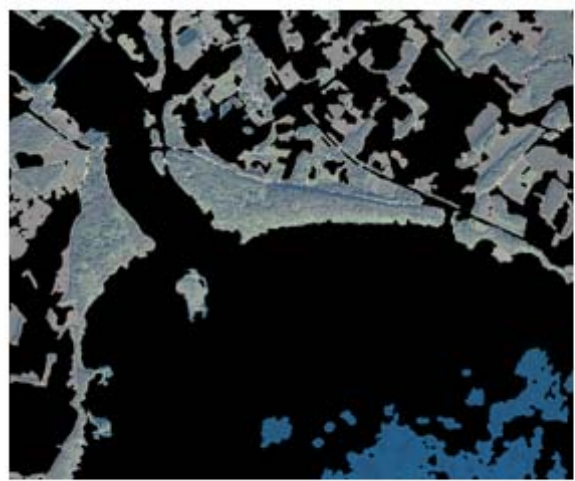

(c) Forest

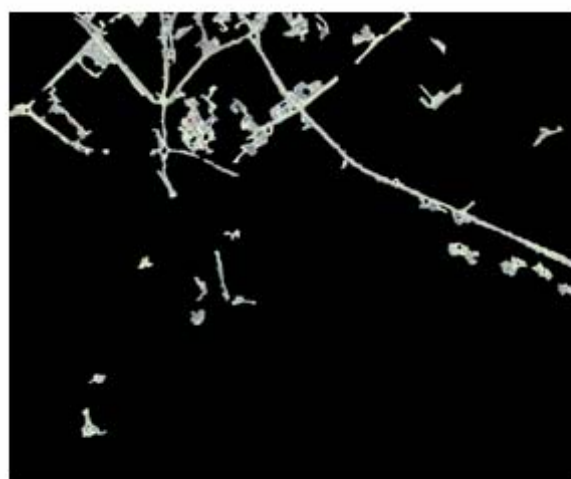

(e) Road

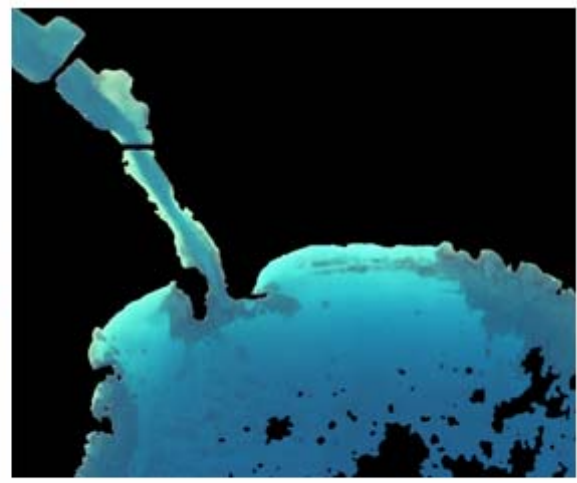

(b) Sea

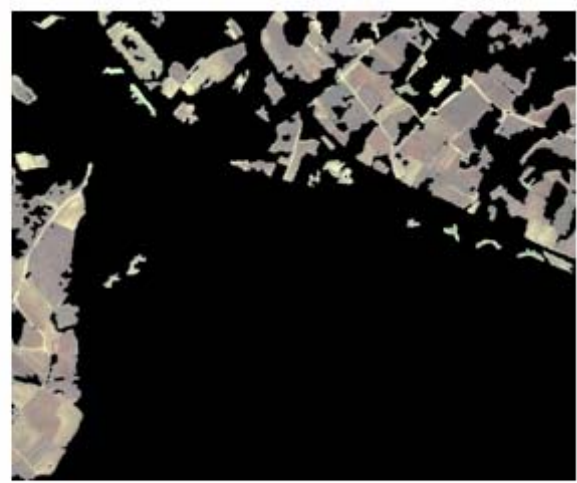

(d) Ground

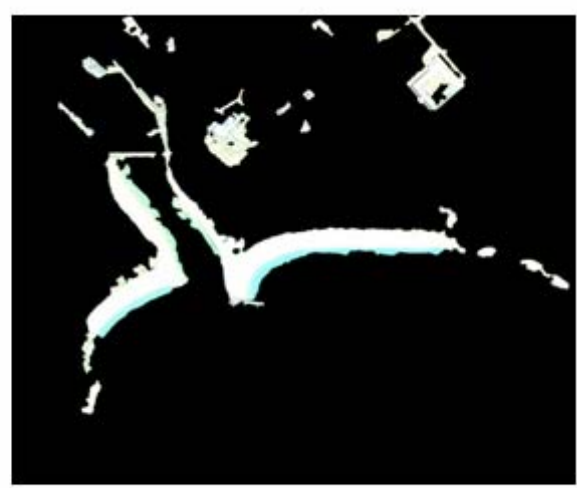

(f) Others

Fig. 5. Region segmentation and categorization results (test image) (IKONOS image: Copyright (C) 2003 Japan Space Imaging Corporation) 


\section{Conclusions}

We proposed a new approach based on supervised locality preserving projections (SLPP) for region segmentation and categorization in high-resolution satellite images. The observed multi-spectral images are first transformed by SLPP and then the transformed spectral components are used as features for classifications. A probabilistic neural network (PNN) is used as a classifier. In this paper, we use only one image as training sample for SLPP subspace learning and classifier (PNN) training. We have shown that it is possible to segment and category other satellite images by using the trained SLPP subspace and PNN.

\section{Acknowledgments}

This work was supported in part by the Strategic Information and Communications R\&D Promotion Program (SCOPE) under the Grand No. 072311002.

\section{References}

1. Avery, T.E., Berlin, G.L.: Fundamentals of Remote Sensing and Airphoto Interpretation. Macmillan Publishing Co., New York (1992)

2. Murai, H., Omatsu, S., OE, S.: Principal Component Analysis for Remotely Sensed Data Classified by Kohonen's Feature Mapping Preprocessor and Multi-Layered Neural Network Classifier. IEICE Trans.Commun. E78-B(12), 1604-1610 (1995)

3. Zeng, X.-Y., Chen, Y.-W., Nakao, Z.: Classification of remotely sensed images using independent component analysis and spatial consistency. Journal of Advanced Computational Intelligence and Intelligent Informatics 8, 216-222 (2004)

4. He, X., Niyogi, P.: Locality Preserving Projections. In: Advances in Neural Information Processing Systems, Vancouver, Canada, vol. 16 (2003)

5. Specht, D.F.: Enhancements to Probabilistic Neural Networks. In: Proceedings of the International Joint Conference on Neural Networks (IJCNN 1992), vol. 1, pp. 761-768 (1992)

6. Chen, Y.-W., Han, X.-H.: Classification of High-Resolution Satellite Images Using Supervised Locality Preserving Projections. In: Lovrek, I., Howlett, R.J., Jain, L.C. (eds.) KES 2008, Part II. LNCS (LNAI), vol. 5178, pp. 149-156. Springer, Heidelberg (2008) 